\title{
CESAM SOLAR MODELS
}

P. MOREL ${ }^{1}$, G. BERTHOMIEU ${ }^{1}$, J. PROVOST ${ }^{1}$

1 Cassini, URA CNRS 1362, Observatoire de la Côte d'Azur, Nice

Y.LEBRETON ${ }^{2}$

${ }^{2}$ DASGAL, URA CNRS 335, Observatoire de Paris, Paris

ABSTRACT Solar models have been computed using the code CESAM constructed at Nice (Morel, 1992), and the new opacities of Livermore for different mixtures. Their global characteristics, predicted capture rates of neutrinos for the chlorine and gallium experiments and their seismological properties are given.

\section{I - GLOBAL PROPERTIES AND NEUTRINO FLUXES}

A set of solar calibrated models has been computed with the CESAM code, insuring the

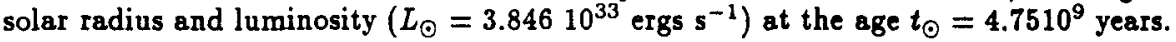

\begin{tabular}{ccccccc} 
TABLE I & & & & & & \\
\hline Models & $\mathrm{Y}$ & $\alpha$ & $\mathrm{T}_{c} 10^{-6}$ & $\rho_{c}$ & ${ }^{37} \mathrm{Cl}$ & ${ }^{71} \mathrm{Ga}$ \\
reference:ST & 0.275 & 1.90 & 15.55 & 151.9 & 7.43 & 127.7 \\
ST1 & 0.276 & 1.89 & 15.51 & 149.6 & 7.19 & 126.5 \\
ST2 & 0.277 & 1.90 & 15.53 & 150.1 & 7.35 & 127.5 \\
ST3 & 0.276 & 1.89 & 15.51 & 149.6 & 6.52 & 124.9 \\
& & & & & & \\
Bahcall et al. & 0.271 & & 15.6 & 148. & 7.9 & 132. \\
Lebreton et al. & 0.278 & 2.16 & 15.54 & 148. & 7.6 & 126.9. \\
Turck Chièze et al. & 0.276 & 1.55 & 15.51 & 147.7 & 5.8 & 125. \\
Sackman et al. & 0.278 & 2.07 & 15.43 & 146.6 & 7.68 & 125. \\
& & & & & & \\
EFF-LAOL & 0.283 & 2.57 & 15.62 & 154.2 & 7.83 & 129.4 \\
CEFFH-LAOL & 0.274 & 2.60 & 15.56 & 151.9 & 7.31 & 126.3 \\
OPAL-RA & 0.284 & 2.56 & 15.68 & 152.5 & 8.43 & 132.2 \\
OPAL-AG & 0.285 & 2.60 & 15.75 & 151.2 & 8.89 & 134.2 \\
OPAL-AGFe (1) & 0.277 & 2.60 & 15.58 & 152.2 & 7.59 & 127.9 \\
OPAL-AGFe (2) & 0.277 & 1.85 & 15.58 & 152.3 & 7.65 & 128.0 \\
OPAL-AG-KU & 0.285 & 1.91 & 15.73 & 151.2 & 8.90 & 134.3 \\
\hline \hline
\end{tabular}

ST: Model with equation of state with the Debye Huckel corrections CEFF and low iron OPAL opacities extended with Kurucr opacities at the surface (KU) and $\mathrm{Z}=$ 0.0194; ST1: Model ST calibrated at a lower age $t_{\odot}=4.610^{9}$ years; ST2: Model ST

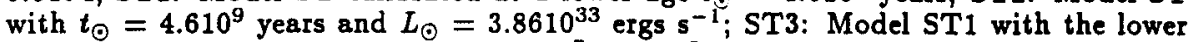
value of the cross section for the reaction ${ }^{7} \mathrm{Be}(\mathrm{p}, \gamma){ }^{8} \mathrm{~B}$ used by Turck-Chièze et al. EFF: Eggleton (et al., 1973) equation of state; LAOL: Los Alamos opacities; OPAL: Livermore opacities with different mixtures: Ross-Aller (RA), Anders and Grevesse mixture with photospheric iron content (AG) and low iron close to the meteoritic abundance (AGFe). 


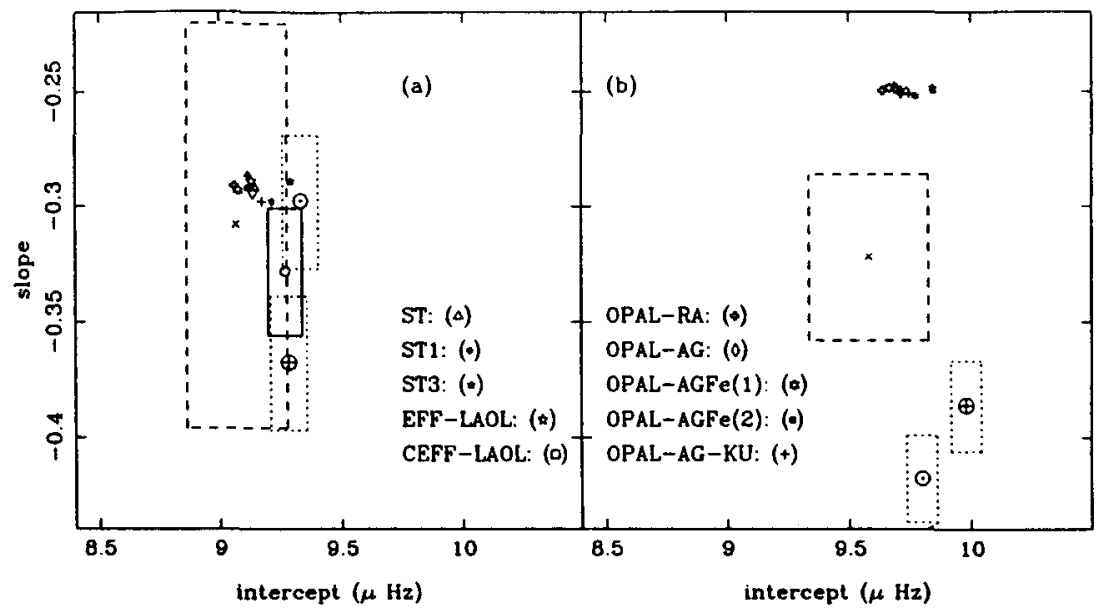

FIGURE I Values of the differences $\nu_{00}-\nu_{02}$ and $\Delta \nu_{0}-\Delta \nu_{2}$ (intercept and slope for $l=0,(\mathrm{a}))$ and $3 / 5\left(\nu_{01}-\nu_{03}\right)$ and $3 / 5\left(\Delta \nu_{1}-\Delta \nu_{3}\right)(3 / 5$ times the intercept and slope for $l=1$, (b)) for different solar models and for the observations of Toutain et al (full line box), Anguera Gubau et al. (two dotted line boxes) corresponding to the solar maximum and minimum and Elsworth et al. (dashed line box).

The comparison of our models with other published models and their sensitivity to the physical inputs (opacities and equation of state) are shown in Table I where the initial helium abundance, mixing length, central temperature and density, capture rates for chlorine and gallium experiments are given. The new OPAL opacity tables are extended to low temperature either by LAOL tables at $T<70000^{\circ} \mathrm{K}$ (except at $\mathrm{T}<$ $600{ }^{\circ} \mathrm{K}$ for OPAL-AGFe(2)) or by Kurucz opacity tables.

Our models are in agreement with the previous solar model computations. They have a higher central density due to the choice of a larger value for the solar age. The neutrino capture rates for chlorine experiment vary by $20 \%$ with the changes in the opacities while they vary by $5 \%$ for the gallium experiment.

\section{II - SEISMOLOGICAL P-MODES PROPERTIES}

Low degree modes give informations on the properties of the core of the Sun. According to the method proposed by Scherrer et al. (1983) the frequencies are fitted to the following polynomial expression:

$$
\nu_{n, l}=\nu_{0}+\Delta \nu_{l}\left(n+\frac{l}{2}-n_{0}\right)+a_{1}\left(n+\frac{l}{2}-n_{0}\right)^{2}
$$

where $n_{0}=21$. The difference $\Delta \nu_{1, l+2}=\nu_{n, l}-\nu_{n-1, l+2}$ is essentially sensitive to the sound velocity in the core of the sun and it is close to a linear function of $n$ characterized by its slope and the intercept ( $\sim$ value for $n=n_{0}$ ).

The values of the slope and the intercept for $\Delta \nu_{0,2}$ and for $\Delta \nu_{1,3}$ of our different models and the observations including an estimation of the observational error bars are plotted in Figure I. The modes with radial nodes between 17 to 26 have been used. The datas of Anguera Gubau et al. are splitted into solar maximum and solar minimum observations. Two different values of the slope and intercept are derived with error boxes which do not overlap. The results of IPHIR are in between which is coherent 
with the dates at which the observations have been made.

The figure shows that the two characteristic parameters derived from the low degree p-modes are close to the observation for $l=0$ and systematically larger for the slope derived from $l=1$ in agreement with Christensen-Dalsgaard (1992) calculations, but they are not much sensitive to the different opacities and equation of state, contrarily to the neutrinos fluxes.

Concerning the modes of intermediate degrees, the new opacities decrease but do not suppress the discrepancy which appears between low $(l<20)$ and high $(l>50)$ degree modes in the difference between observed and theoretical frequencies. The frequencies with degree ranging from 20 to 50 correspond to the modes having their turning point just below and just above the convection zone and the persistency of this observed discrepancy may be the signature of the presence of some penetration taking place at the lower boundary of the convection zone.

In conclusion the global properties of our models are in agreement with the previous results. The causes of the differences are pointed out in a more complete discussion in Berthomieu et al. (1992). With the new opacities of Livermore with low photospheric iron content we find for the chlorine experiment $\Phi_{{ }^{3} \mathrm{Cl}}=7.43 \pm 2.7 \mathrm{SNU}$ far from the measure of Davis and for the gallium experiment $\Phi_{7{ }_{1 a}}=127.7 \pm 20 \mathrm{SNU}$.

\section{REFERENCES}

Anders E., Grevesse N. 1989, Geochim. Cosmochim. Acta 53, 197

Anguera Gubau M., Palle P., Perez Hernandez F., Regulo C. Roca Cortes T. 1992, Astron. Astrophys. 255 363-372

Bahcall J., Ulrich R.K. 1988, Rev. Mod. Physics 60, 296

Berthomieu G., Provost J., Morel P., Lebreton Y. 1992, submitted to Astron. Astrophys.

Christensen-Dalsgaard J. 1992, Challenges to Theories of the Structure of Mode rate-Mass Stars, D.O. Gough, J. Toomre (Eds), Springer Verlag, p 11-36

Eggleton P.P., Faulkner J., Flannery B.P. 1973, Astron. Astrophys. 23 325-330

Elsworth Y., Howe R., Isaak G.R., McLeod C.P., New R. 1990, Nature 345, 322-324

Huebner, W.F., Merts, A.L., Magee, N.H.Jr, Argo, M.F. 1977, Astrophysical Opacity Library, Los Alamos Scientific Laboratory Report LA-6760-M

Iglesias C. A., Rogers F. J. 1991, A strophys. J. 371, 408-417

Lebreton Y., Däppen W. 1998, Seismology of the Sun \& Sun-like Starg' ed. E.J. Rolfe, ESA SP-286, pp 661-664

Libbrecht K.G., Woodard M.F., Kaufman J.M. 1990, Astrophys. J. Supp. 74, 1129

Morel P., 1992, these proceedings

Ross J.E., Aller L.H. 1976, Science 181, 1223

Sackmann I.J., Boothroyd A.I., Fowler W.A. 1990 Astrophys. J. 360, 727-736

Scherrer P.H., Wilcox J., Christensen-Dalsgaard J. Gough D.O. 1983 Solar Phys. 82, 75-87

Toutain Th., Fröhlich C. 1992, Astron. Astrophys. to appear

Turck-Chièze S., Cahen S., Cassé M., Doom C. 1988, Astrophys. J. 335, 415 